\title{
Efficacy and Safety of Clarithromycin as Treatment for Mediterranean Spotted Fever in Children: A Randomized Controlled Trial
}

\section{Antonio Cascio, ${ }^{1}$ Claudia Colomba,' Domenico Di Rosa, Ludovico Salsa, ${ }^{1}$ Lucio di Martino, ${ }^{2}$ and Lucina Titone ${ }^{1}$}

"Istituto di Patologia Infettiva e Virologia, Ospedale "G. Di Cristina," Università di Palermo, Palermo, and ${ }^{2} U n i t a$ Operativa di Pediatria Infettivologica, Ospedale Pausillipon, Azienda Ospedaliera Santobono-Pausillipon, Naples, Italy

Fifty-one children with Mediterranean spotted fever (MSF) were randomized to receive either clarithromycin, $15 \mathrm{mg} / \mathrm{kg} /$ day orally in 2 divided doses, or chloramphenicol, $50 \mathrm{mg} /$ $\mathrm{kg} / \mathrm{day}$ orally in 4 divided doses, for 7 days. Mean time to defervescence was $36.7 \mathrm{~h}$ in the clarithromycin group and $47.1 \mathrm{~h}$ in the chloramphenicol group $(P=.047)$. Clarithromycin could be an acceptable therapeutic alternative to chloramphenicol and to tetracyclines for children aged $<8$ years with MSF.

Mediterranean spotted fever (MSF) is caused by Rickettsia conorii and transmitted by the dog tick Rhipicephalus sanguineus. It is an acute infectious disease typically characterized by fever, skin rash, and a black scar at the site of a tick bite ("tache noire"). Three hundred cases are reported every year (mainly from June through September) on the Italian island of Sicily [1]. In North America MSF is the most frequently imported variety of rickettsiosis [2].

$R$. conorii is an obligate intracellular bacterium, and the main feature of MSF is a generalized vasculitis in which $R$. conorii are localized within the endothelial cells [3]. Effective intracellular antimicrobial concentrations are considered the sine qua non of a successful treatment for this disease. The macrolides chloramphenicol and doxycycline penetrate eukaryotic

Received 23 October 2000; revised 17 January 2001; electronically published 21 June 2001 Informed consent was obtained from the parents of all patients, and the guidelines of the G. Di Cristina Hospital were followed in the conduct of the clinical research.

Reprints or correspondence: Prof. Antonio Cascio, Istituto di Patologia Infettiva e Virologia, Ospedale "G. Di Cristina," Università di Palermo, Piazza Montalto n. 8, 90134 Palermo, Italia (acascio@unime.it), or Prof. Claudia Colomba (same address; claudia.colomba@libero.it).

Clinical Infectious Diseases 2001;33:409-11

(C) 2001 by the Infectious Diseases Society of America. All rights reserved. $1058-4838 / 2001 / 3303-0026 \$ 03.00$ membranes and concentrate within the cytoplasm of phagocytic cells.

Standard treatment for MSF is the oral or parenteral administration of tetracyclines or chloramphenicol. However, both of these drugs have significant adverse effects, especially in children. Tetracyclines can cause staining of the teeth and bone toxicity; chloramphenicol can cause gray-baby syndrome, aplastic anemia, and acute hemolytic anemia in patients with the Mediterranean form of glucose-6-phosphate dehydrogenase (G6PD) deficiency. During the past 10 years, we have regarded chloramphenicol as "the lesser of two evils" and have used this antibiotic as the drug of choice for treatment of MSF in children [1].

The macrolides clarithromycin, azithromycin, roxithromycin, and josamycin, which are often used to treat infections in children, lack such adverse effects and seem to be effective in vitro against $R$. conorii [4-6]. Recently, 14 hydroxy-clarithromycin, the primary metabolite of clarithromycin, has been demonstrated to attain serum concentrations 3 times greater than its MIC $[4,5]$. Given this background information, we thought it appropriate to undertake a randomized clinical trial comparing the efficacy and safety of clarithromycin versus chloramphenicol as treatment for MSF in children.

Patients and methods. All patients with clinically suspected MSF who were admitted to the "G. Di Cristina" children's hospital in Palermo, Italy, from June through September 1998 were evaluated for possible inclusion in the study. At admission, a complete physical examination and laboratory tests were performed. Indirect immunofluorescence assay for $R$. conorii was performed using a commercial kit (Rickettsia Conofi-Spot IF; bioMerieux).

Exclusion criteria were as follows: age $>14$ years, lack of parental informed consent, history of allergy to the drugs being studied, severe disease (e.g., neurological signs, hemorrhagic manifestations, or sepsis), renal or liver insufficiency, immunodeficiency, G6PD deficiency, or previous treatment with antibiotic agents potentially active against Rickettsia species in the 7 days before hospitalization.

After informed consent was obtained, children with clinically suspected MSF and without exclusion criteria were randomly assigned to receive 7 days of treatment with either clarithromycin (Abbott), $15 \mathrm{mg} / \mathrm{kg} /$ day orally in 2 divided doses, or chloramphenicol (Pharmacia), $50 \mathrm{mg} / \mathrm{kg} /$ day orally in 4 divided doses. Patients were randomized to 1 of the 2 treatment groups by means of a list of computer-generated random numbers. The final study population was the group of enrolled children 
Table 1. Baseline characteristics of the $\mathbf{5 1}$ patients with Mediterranean spotted fever, according to drug treatment group.

\begin{tabular}{|c|c|c|c|}
\hline \multirow[b]{2}{*}{ Characteristic } & \multicolumn{2}{|c|}{ Drug treatment group } & \multirow[b]{2}{*}{$P$} \\
\hline & $\begin{array}{l}\text { Chloramphenicol } \\
\qquad(n=26)\end{array}$ & $\begin{array}{c}\text { Clarithromycin } \\
\quad(n=25)\end{array}$ & \\
\hline Male sex & 14 & 16 & NS \\
\hline Age, years ${ }^{a}$ & $5.2 \pm 2.6$ & $7.1 \pm 3.6$ & .035 \\
\hline Fever & 26 & 25 & NS \\
\hline $\begin{array}{l}\text { Duration of fever before } \\
\text { treatment, mean days }\end{array}$ & $3 \pm 1.3$ & $3.3 \pm 1.7$ & NS \\
\hline Exanthema & 26 & 25 & NS \\
\hline Tache noire & 26 & 25 & NS \\
\hline $\mathrm{ESR}, \mathrm{mm} / \mathrm{h}$ & $22.5 \pm 9.2$ & $27.9 \pm 11.7$ & NS \\
\hline AST level, IU/L & $57.3 \pm 25$ & $40.2 \pm 18.8$ & NS \\
\hline WBC count, cells $\times 10^{3} / \mathrm{mL}$ & $6077 \pm 2464$ & $6954 \pm 2745$ & NS \\
\hline Platelet count, cells $\times 10^{3} / \mathrm{mL}$ & $214 \pm 57.4$ & $185 \pm 56.7$ & NS \\
\hline
\end{tabular}

NOTE. Data are no. of patients or mean \pm SD of the specified unit. ESR, erythrocyte sedimentation rate; AST, aspartate aminotransferase; tache noire, a black scar at the site of a tick bite; NS, not significant.

a There were no significant differences between the 2 groups with respect to characteristics at presentation (by Student $t$ test or $\chi^{2}$ test), except age.

who had a confirmed diagnosis of MSF. Diagnosis was considered confirmed if the patient had a score $>25$ for the MSF diagnostic test described by Raoult et al. [7].

Clinical response to antibiotic treatment was evaluated on the basis of the pattern of body temperature and the improvement of the clinical signs and symptoms of the disease. The end point was defined as the first achievement of persistent defervescence, which was defined as an axillary temperature $\leqslant 37^{\circ} \mathrm{C}$ for at least 3 consecutive days. Nurses and parents were instructed to give paracetamol, $10 \mathrm{mg} / \mathrm{kg}$, every time a patient's temperature was $>38.5^{\circ} \mathrm{C}$, but not $>4$ times per day. If the patient was discharged before day 7 of the hospitalization, the parents were told to check the child's temperature at least twice daily, to call us if fever or other problems reappeared, and to continue to administer the assigned treatment to complete the 7-day regimen.

The data were analyzed using StatSoft software (Statistica). Contingency data were analyzed by 2 -tailed $\chi^{2}$ test or Fisher's exact test, and continuous data were analyzed by use of Student $t$ test. The Pearson correlation coefficient was computed to verify the existence of correlations between variables. A 2-sided $P$ value $<.05$ was considered significant for all analyses.

Results. Seventy-five patients with suspected MSF were admitted to the hospital during the study period. Seven patients who had received drugs that are potentially active against Rickettsia species during the 7 days preceding the study were excluded from the study. Sixty-eight patients were randomized to receive the study drugs. Fifty-one of these 68 patients had an MFS diagnostic score $>25$ and comprised the final study population; 26 had been assigned to receive chloramphenicol and 25 to receive clarithromycin. The baseline characteristics of these patients are summarized in table 1; the patients in the 2 treatment groups were similar with respect to major demographic variables and characteristics of MSF at study enrollment except that the mean age of the clarithromycin group was 2 years greater than that of the chloramphenicol group $(P=$ .035; $\chi^{2}$ test).

All patients received the assigned therapy for 7 days and had recovered by the end of therapy. Defervescence was achieved at a mean duration $\pm \mathrm{SD}$ of $36.7 \pm 18.1 \mathrm{~h}$ (range, 3-84 h) in the clarithromycin group and $47.1 \pm 21.9 \mathrm{~h}$ (range, 12-96 h) in the chloramphenicol group; these differences were statistically significant $(t=2.14 ; P=.047)$. There was no significant difference between the 2 groups with respect to the use of paracetamol (used by all patients), nonsteroidal anti-inflammatory drugs (used by none of the patients), or corticosteroids (used by 3 patients in the clarithromycin group and by 2 in the chloramphenicol group; $P>.20$ for all 3 comparisons). The median duration of exanthema after the beginning of therapy was 3 days (range, 1-5 days) for both groups. To exclude the possibility that the more rapid defervescence in the clarithromycin group was due to the greater mean age of these patients, a Pearson correlation coefficient was computed. A correlation between these 2 variables was excluded ( $r=.04 ; P=.7)$.

No relapses were observed during 30 days of follow-up. In all patients, a 4 -fold increase in the antibody titer to $R$. conorii was observed on day 30. Clarithromycin and chloramphenicol were equally well tolerated and no major side effects were ob- 
served; vomiting was reported for 2 patients treated with clarithromycin and for 1 treated with chloramphenicol. None of the patients required discontinuation of the drugs.

Discussion. Until now there has not been a gold-standard therapy for children with rickettsial diseases. The few studies that have evaluated the in vitro activity of macrolides against R. conorii [4-6] suggest that clarithromycin therapy may be of interest because its pharmacological and pharmacokinetic properties are possibly superior to those of other macrolides [5]. Moreover, the combination of clarithromycin and its 14hydroxy metabolite produce MICs and postantibiotic effects that are greater than those associated with use of clarithromycin alone [4].

Extracellular antimicrobial concentrations may not be adequate to eliminate intracellular pathogens, especially for some of the new macrolides that exhibit a large volume of distribution into cells and tissue [4]. Until future studies determine the true intracellular (endothelial) MIC and maximum concentration for macrolide treatment of infection with Rickettsia species, we must continue to rely on determination plasma concentrations and assume that adequate phagocytic membrane penetration occurs. Because in vitro antimicrobial data cannot demonstrate a direct clinical correlation to the selection and use of antimicrobial pharmacotherapy, animal and human clinical trials are needed to determine the specific role that these agents may play in the treatment of these infections [5].

To the best of our knowledge, no other studies have been conducted on the use of clarithromycin therapy to treat MSF. Three randomized clinical trials have been performed to evaluate macrolides as treatment for MSF. The first of these trials compared 10 days of treatment with tetracycline hydrochloride (10 mg/kg q.i.d.) with 10 days of treatment with erythromycin stearate (12.5 mg/kg q.i.d.) in 81 children [8]. The second study compared 5 days of treatment with doxycycline $(5 \mathrm{mg} / \mathrm{kg}$ once daily) with 3 days of treatment with azithromycin $(10 \mathrm{mg} / \mathrm{kg}$ once daily) in 30 children [9]. The third study compared 1 day of treatment with doxycycline $(2.5 \mathrm{mg} / \mathrm{kg}$ b.i.d.) with 5 days of treatment with josamycin ( $25 \mathrm{mg} / \mathrm{kg}$ b.i.d.) in 27 adults and 32 children [10]. The first trial showed that the patients treated with tetracycline hydrochloride had more rapid disappearance of fever and clinical symptoms than did patients treated with erythromycin [8]. However, the in vitro activity of erythromycin against $R$. conorii is not as potent as that of newer macrolides, such as clarithromycin. The second trial showed that azithromycin and doxycycline have equal efficacy [9]. In the third trial, no statistically significant differences were observed between the doxycycline group and the josamycin group [10]. Both of the latter 2 studies enrolled small numbers of children; however, the clinical equivalence of tetracyclines and the newer macrolides in the small number of patients assessed is noteworthy.

In our study, the patients treated with clarithromycin defervesced more rapidly than did those treated with chloramphenicol. It is the largest randomized clinical study yet performed to assess the newer macrolides as treatment for rickettsial infections in children. Clarithromycin could constitute an acceptable therapeutic alternative to chloramphenicol and to tetracyclines for children aged $<8$ years who have MSF.

\section{Acknowledgments}

We thank Prof. D. Raoult for having reviewed the manuscript and Prof. D. Paterson and Prof. S. Antinori for critical advice.

\section{References}

1. Cascio A, Dones P, Romano A, et al. Clinical and laboratory findings of boutonneuse fever in Sicilian children. Eur J Pediatr 1998; 157:482-6.

2. Raoult D, Roux V. Rickettsioses as paradigms of new emerging infectious diseases. Clin Microbiol Rev 1997; 10:694-719.

3. McDonald JC, MacLean JD, McDade JE Imported rickettsial disease: clinical and epidemiological features. Am J Med 1988; 85:799-805.

4. Ives TJ, Marston EL, Regnery RL, et al. In vitro susceptibilities of Rickettsia and Bartonella spp. to 14- hydroxy-clarithromycin as determined by immunofluorescent antibody analysis of infected Vero cell monolayers. J Antimicrob Chemother 2000; 45:305-10.

5. Ives TJ, Manzewitsch P, Regnery RL, et al. In vitro susceptibilities of Bartonella henselae, B. quintana, B. elizabethae, Rickettsia rickettsii, $R$. conorii, R. akari, and $R$. prowazekii to macrolide antibiotics as determined by immunofluorescent-antibody analysis of infected Vero cell monolayers. Antimicrob Agents Chemother 1997; 41:578-82.

6. Rolain JM, Maurin M, Vestris G, et al. In vitro susceptibilities of 27 Rickettsiae to 13 antimicrobials. Antimicrob Agents Chemother 1998; 42:1537-41.

7. Raoult D, Tissot-Dupont H, Caraco P, et al. Mediterranean spotted fever in Marseille: descriptive epidemiology and the influence of climatic factors. Eur J Epidemiol 1992; 8:192-7.

8. Munoz-Espin T, Lopez-Pares P, Espejo-Arenas E, et al. Erythromycin versus tetracycline for treatment of Mediterranean spotted fever. Arch Dis Child 1986; 61:1027-9.

9. Meloni G, Meloni T. Azithromycin vs doxycycline for Mediterranean spotted fever. Pediatr Infect Dis J 1996; 15:1042-4.

10. Bella F, Font B, Uriz S, et al. Randomized trial of doxycycline versus josamycin for Mediterranean spotted fever. Antimicrob Agents Chemother 1990; 34:937-8. 